\title{
Zwei Wege zu Si-funktionellen Cyclosilazanen - Kristallstruktur des 1,3,6,8,10,12-Hexa-aza-2,4,5,7,9,11-hexa- sila-dispiro[4.1.4.1]dodecan
}

\author{
Susanne Bartholmet, Uwe Kuingebiel**, George M. Sheldrick und \\ Dietmar Stalke
}

Göttingen, Institut für Anorganische Chemie der Universität

Inhaltsübersicht. Aminochlorsilane $\left[\mathrm{RSiCl}_{2} \mathrm{NHCMe}_{3}, \mathrm{R}=\mathrm{Cl}(\mathbf{1}), \mathrm{H}(\mathbf{2})\right]$ werden durch die Reaktion der Chlorsilane mit LiNHCMe $e_{3}$ erhalten. $\mathrm{HSiCl}_{2} \mathrm{~N}$ (iso-Bu)SiMe ${ }_{3}$ (3) entsteht in der Reaktion von $\mathrm{HSiCl}_{3}$ mit $\mathrm{LiN}\left(\right.$ iso-Bu) $\mathrm{SiMe}_{3} . \mathrm{HSiCl}_{3}$ reagiert mit $\mathrm{LiN}\left(\mathrm{CMe}_{3}\right) \mathrm{SiMe}_{3}$ unter $\mathrm{LiCl}$ - und $\mathrm{Me}_{3} \mathrm{SiCl}$ Abspaltung zum Cyclodisilazan [( $\left.\mathrm{HSiCl}-\mathrm{NCMe}_{3}\right)_{2}$ (4)]. Neben $\mathrm{C}_{6} \mathrm{H}_{5} \mathrm{SiCl}_{2} \mathrm{~N}\left(\mathrm{CMe}_{3}\right) \mathrm{SiMe}_{3}$ (5) ist $\mathrm{C}_{6} \mathrm{H}_{5} \mathrm{SiCl}_{2} \mathrm{NHCMe}_{3}(6)$ das Hauptprodukt der Reaktion von Trichlorphenylsilan mit LiN( $\left.\mathrm{CMe}_{3}\right) \mathrm{SiMl}_{3}$. 3 verliert thermisch $\mathrm{Me}_{3} \mathrm{SiCl}$. Es entsteht das Cyclotrisilazan [(HSiCl-N-iso-Bu $)_{3}$ (7)]. 5 verliert thermisch iso-Butan unter Bildung von $\mathrm{C}_{6} \mathrm{H}_{5}-\mathrm{SiCl}_{2}-\mathrm{NH}-\mathrm{SiMe}_{3}(\mathbf{8})$. 1, 2 und 6 reagieren mit n- $\mathrm{C}_{6} \mathrm{H}_{9} \mathrm{Li}$ unter Butan- und LiCl-Abspaltung zu den Cyclodisilazanen $\left[\left(\mathrm{RSiCl}-\mathrm{NCMe}_{3}\right)_{2}, \mathbf{R}=\mathbf{H}(\mathbf{4})\right.$, $\mathrm{Cl}(9), \mathrm{C}_{6} \mathrm{H}_{5}(\mathbf{1 0}) .4$ wird durch $\mathrm{NaF}$ zu $\left(\mathrm{HFSi}-\mathrm{NCMe}_{3}\right)_{2}$ (11) fluoriert. Die Alkoholyse von 4 führt zur Bildung von $\left[\left(\mathrm{H}(\mathrm{RO}) \mathrm{Si}-\mathrm{NCMe}_{3}\right)_{2}, \mathrm{R}=\mathrm{Me}(\mathbf{1 2}), \mathrm{C}_{6} \mathrm{H}_{5}(\mathbf{1 3})\right.$, die Aminolyse zu $\left[\left(\mathrm{H}\left(\mathrm{R}_{2} \mathrm{~N}\right) \mathrm{Si}-\mathrm{NCMe}_{3}\right)_{2}\right.$, $\mathrm{R}=\mathrm{Me}(\mathbf{1 4}), \mathrm{C}_{2} \mathrm{H}_{5}$ (15). Nur ein Chloratom von 4 wird in der Reaktion mit $\mathrm{H}_{2} \mathrm{NCMe}_{3}$ substituiert (16). Mit Lithium reagiert 4 zum 1,3,6,8,10,12-Hexa-aza-2,4, $5,7,9,11$-hexasila-dispiro[4.1.4.1]dodecan (17). Die Kristallstruktur von 17 wird mitgeteilt.

Two Ways to Si-functional Cyclosilanes - Crystal Structure of 1,3,6,8,10,12-Hexa-aza $2,4,5,7,9,11$-hexasila-dispiro[4.1.4.1] dodecan

Abstract. Aminochlorosilanes $\left[\mathrm{RSiCl}_{2} \mathrm{NHCMe}_{3}, \mathrm{R}=\mathrm{Cl}(\mathbf{1}), \mathrm{H}(\boldsymbol{(})\right)$ are obtained in the reaction of the chlorosilanes with $\mathrm{LiNHCMe}_{3}$. $\mathrm{HSiCl}_{2} \mathrm{~N}$ (iso- $\mathrm{Bu}$ ) $\mathrm{SiMe}_{3}(3)$ is formed in the reaction of $\mathrm{HSiCl}_{3}$ and $\mathrm{LiN}$ (iso-Bu) $\mathrm{SiMe}_{3} . \mathrm{HSiCl}_{3}$ reacts with $\mathrm{LiN}\left(\mathrm{CMe}_{3}\right) \mathrm{SiMe}_{3}$ under $\mathrm{LiCl}$ and $\mathrm{Me}_{3} \mathrm{SiCl}$ elimination to give the cyclodisilazane $\left[\left(\mathrm{HSiCl}-\mathrm{NCMe}_{3}\right)_{2}\right.$ (4)]. In addition to $\mathrm{C}_{6} \mathrm{H}_{5} \mathrm{SiCl}_{2} \mathrm{~N}\left(\mathrm{CMe}_{3}\right) \mathrm{SiMe}_{3}$ (5), the main product of the reaction of trichloro-phenylsilane with $\mathrm{LiN}\left(\mathrm{CMe}_{3}\right) \mathrm{SiMe}_{3}$ is $\mathrm{C}_{6} \mathrm{H}_{5} \mathrm{SiCl}_{2} \mathrm{NHCMe}_{3}(6) .3$ loses $\mathrm{Me}_{3} \mathrm{SiCl}$ thermally, giving the cyclotrisilazane $\left[(\mathrm{HSiCl}-\mathrm{N}-\mathrm{iso}-\mathrm{Bu})_{3}\right.$ (7)]. 5 loses iso-butane thermally with formation of $\mathrm{C}_{6} \mathrm{H}_{5}-\mathrm{SiCl}_{2}-\mathrm{NH}-\mathrm{SiMe}_{3}(\mathbf{8}) .1,2$ and 6 react with $\mathrm{LiC}_{4} \mathrm{H}_{9}$ under butane and $\mathrm{LiCl}$ elimination to give the cyclodisilazanes $\left[\left(\mathrm{RSiCl}-\mathrm{NCMe}_{3}\right)_{2}, \mathrm{R}=\mathrm{H}(\mathbf{4}), \mathrm{Cl}(\mathbf{9}), \mathrm{C}_{6} \mathrm{H}_{5}(\mathbf{1 0})\right]$. 4 is fluorinated to $\left(\mathrm{HSiF}-\mathrm{NCMe}_{3}\right)_{2}(11)$ by $\mathrm{NaF}$. The alcoholysis of 4 leads to the formation of $\left[\left(\mathrm{H}(\mathrm{RO}) \mathrm{Si}-\mathrm{NCMe}_{3}\right)_{2}, \mathrm{R}=\mathrm{Me}(\mathbf{1 2}), \mathrm{C}_{6} \mathrm{H}_{5}(\mathbf{1 3})\right]$, the aminolysis to $\left[\left(\mathrm{H}\left(\mathrm{NR}_{2}\right) \mathrm{Si}-\mathrm{NCMe}_{3}\right)_{2}, \mathrm{R}=\mathrm{Me}(\mathbf{1 4})\right.$, $\mathrm{C}_{2} \mathrm{H}_{5}$ (15), only one chloro atom of 4 is substituted in the reaction with $\mathrm{H}_{2} \mathrm{NCMe}_{3}(\mathbf{1 6})$. 4 reacts with lithium to give the $1,3,6,8,10,12$-hexa-aza-2, 4, 5, 7,9,11-hexasila-dispiro[4.1.4.1]dodecgn (17), for which the crystal structure is reported.

Die Reaktion von Dichlorsilanen mit $\mathrm{NH}_{3}$ ist eine klassische Methode der Cyclosilazan-Synthese [1]. Sie führt zu NH-funktionellen Ringen. Primäre Amine mit kleinen Alkylgruppen reagieren mit Dichlordimethylsilan zu Alkylamino- 
dimethylchlorsilanen, die bei Raumtemperatur instabil sind und sich weitgehend zu Alkylammoniumchloriden, 1,3-Dichlor-2-alkyl-tetramethyldisilazanen und 1-Chlor-3-alkylamino-2-alkyltetramethyldisilazanen zersetzen [2]. Mit zunehmender Substituentengröße werden Chlorsilylverbindungen primärer Amine kinetisch stabilisiert, so daß sie zur Darstellung Cl-funktioneller Cyclosilazane genutzt werden können.

Die beim Nachbarelement Phosphor in Reaktionen der Halogenide mit Alkalisalzen der Silylamine häufig mit der Salzabspaltung verbundene Halogentrimethylsilylabspaltung führt je nach Stöchiometrie zur Bildung von (PN)-Ringen oder Iminophosphanen [3]. In der auf Halogensilane übertragenen Reaktion erfolgt weitgehend nur die Salzeliminierung. Es entstehen die acyclischen Verbindungen [3]. Mit zunehmender Größe des Amines wird jedoch auch Halogensilanabspaltung beobachtet, $\mathbf{d}$. h. das primär gebildete (Trimethylsilylamino)chlorsilan wird in diesem Fall sterisch destabilisiert, es entstehen die entsprechenden (SiN)-Ringe [4].

In dieser Arbeit werden zwei Wege für die Synthese Si-funktioneller (SiN)Ringe vorgestellt und deren Reaktionsverhalten untersucht.

\section{Ergebnisse}

Im Gegensatz zur bekannten Aminolyse von Tri- oder Tetrachlorsilanen durch primäre Amine, die unter vollständiger Chlorsubstitution und/oder der Kondensation zu Cyclosilazanen verläuft, reagiert lithiiertes tert-Butylamin mit $\mathrm{HSiCl}_{3}$ bzw. $\mathrm{SiCl}_{4} \mathrm{zu}$ den Aminodi- bzw. Aminotrichlorsilanen 1 und 2 (Gl. (1)).

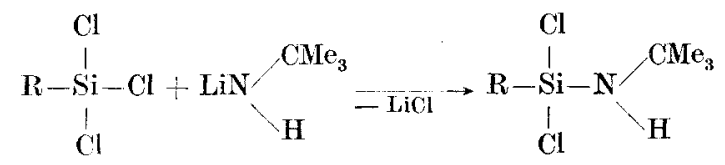

$$
1,2
$$

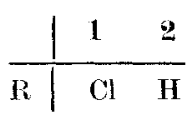

1 und 2 sind farblose Flüssigkeiten, die wider Erwarten keine Neigung zur Kondensatior zeigen.

1977 wurde erstmals die thermische Halogentrimethylsilan-Abspaltung aus Trimethylsilylamino-chlorsilanen zur Darstellung Si-funktioneller Cyclosilazane genutzt [4]. Die Substitutionsverbindungen sind zum Teil bereits derart instabil, daß sie im Gegensatz zu den NH-Verbindungen wie 2 nicht rein isoliert werden können. $\mathrm{HSiCl}_{3}$ reagiert $\mathrm{z}$. B. mit lithiiertem iso-Butyltrimethylsilylamin zu 3 
und mit lithiiertem tert-Butyltrimethylsilylamin zum Vierring 4 (G1. (2)) .

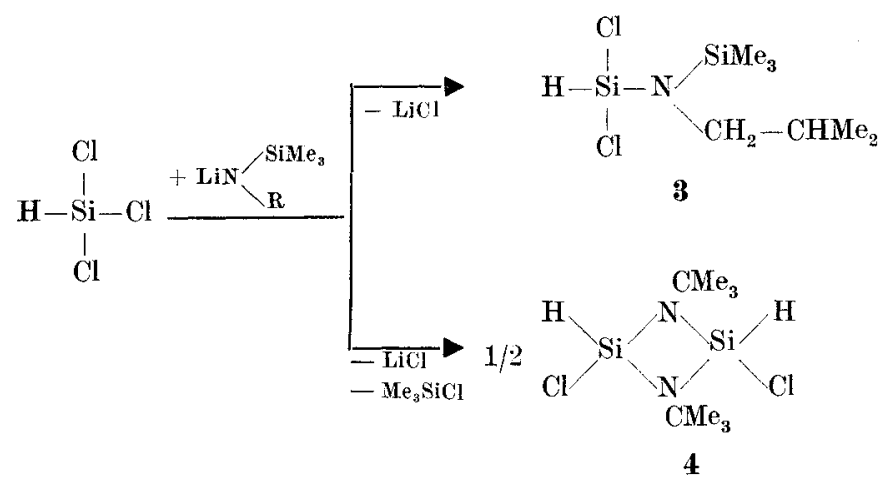

4 entsteht als destillativ nicht trennbares cis/trans-Isomerengemisch im Verhältnis 1:2. Außer vom Trimethylsilylamin ist die Produktbildung vom reagierenden Chlorsilan abhängig. $\mathrm{MeSiCl}_{3}$ reagiert analog $\mathrm{HSiCl}_{3}$ mit $\mathrm{LiN}\left(\mathrm{CMe}_{3}\right) \mathrm{SiMe}_{3}$ in THF zum Cyclodisilazan, $\mathrm{C}_{6} \mathrm{H}_{5} \mathrm{SiCl}_{3}$ zur Substitutionsverbindung 5 als Nebenprodukt und zur NH-Verbindung 6 alsHauptprodukt (Gl. (3)).

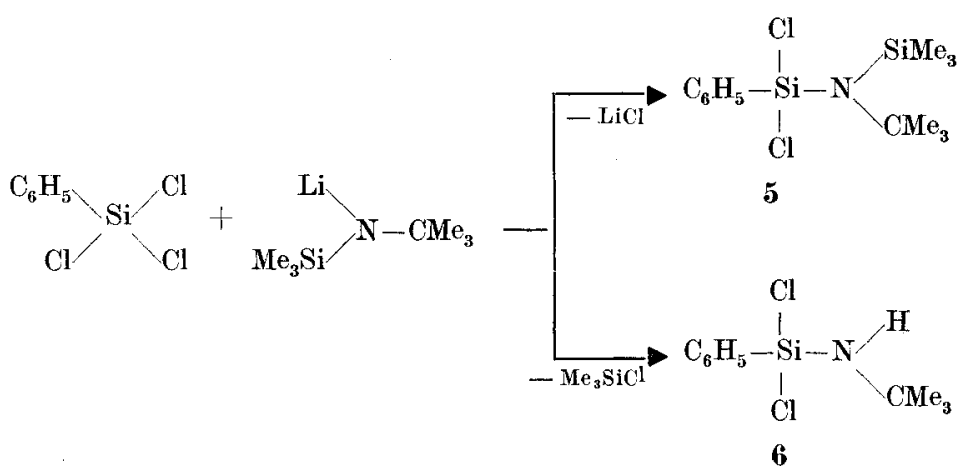

6 entsteht unter $\mathrm{Me}_{3} \mathrm{SiCl}-\mathrm{Abspaltung}$ [5]. Der Aminwasserstoff wird vom Lösungsmittel geliefert.

Wird 3 bei Normaldruck auf etwa $100^{\circ} \mathrm{C}$ erhitzt, tritt $\mathrm{Me}_{3}$ SiCl-Abspaltung unter Bildung des sechsgliedrigen Ringes 7 ein (G1. (4)). Aluminiumtrichlorid katalysiert die $\mathrm{Me}_{3} \mathrm{SiCl}$-Abspaltung. Die Ringgröße wird beeinflußt vom Raumbedarf der Organylgruppe des Amins. Die tert-Butylgruppe verursacht die Bildung von Cyclodisilazanen (Gl. (2)).

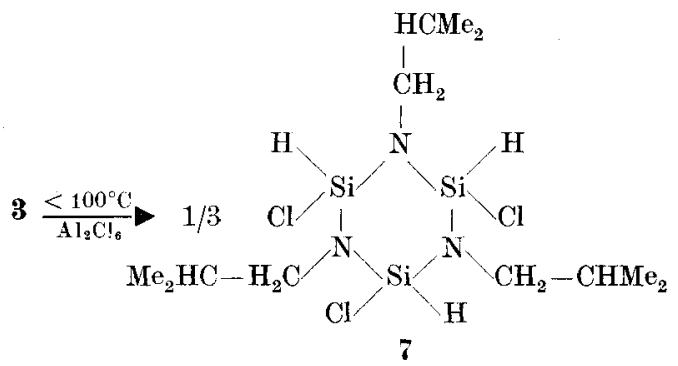


5 verliert thermisch iso-Buten. Es entsteht 8.

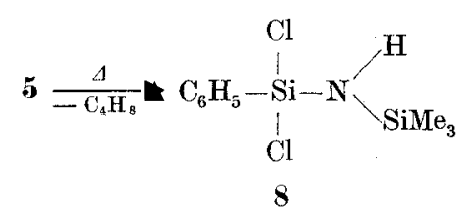

Für die Darstellung Si-funktioneller Cyclosilazane ist neben der Halogensilan-Abspaltung die Salzeliminierung die Methode der Wahl. 1, 2 bzw. 6 reagieren mit $\mathrm{C}_{4} \mathrm{H}_{9} \mathrm{Li}$ zu $\mathrm{C}_{4} \mathrm{H}_{10}, \mathrm{LiCl}$ und den Vierringen 4, 9 und 10 (Gl. (6)).

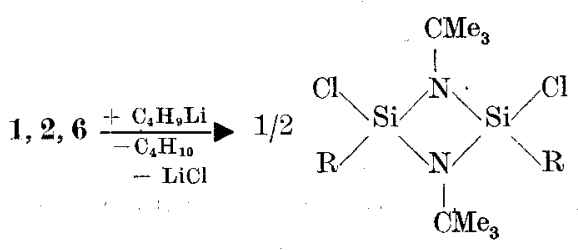

$$
\begin{aligned}
& 4,9,10 \\
& \begin{array}{l|lll} 
& \mathbf{4} & \mathbf{9} & \mathbf{1 0} \\
\hline \mathrm{R} & \mathrm{H} & \mathrm{Cl} & \mathrm{C}_{\mathbf{6}} \mathrm{H}_{5}
\end{array}
\end{aligned}
$$

Das Schema 1 zeigt einige einfache Reaktionen, die am Cyclodisilazan 4 unter Erhalt des Vierringsystems durchgeführt wurden.

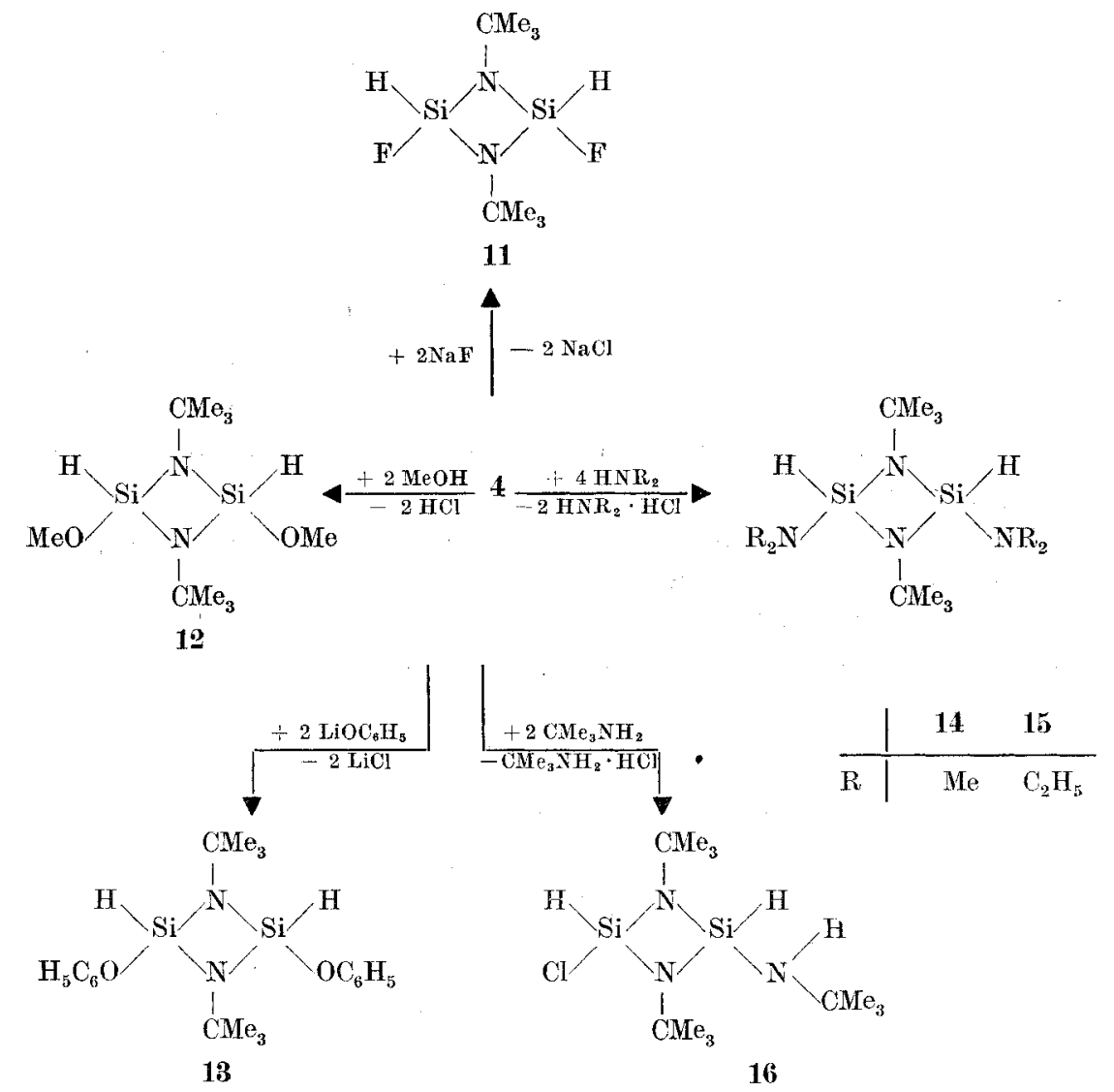


Die Chloratome werden durch Fluoratome in der Reaktion von 4 mit $\mathrm{NaF}$ in $\mathrm{CH}_{3} \mathrm{CN}$ ersetzt. Durch Methanolyse entsteht 12 und in der Reaktion mit Lithiumphenolat13. Beide Chloratome von 4 werden durch Aminolyse von 4 mit Dimethylund Diethylamingruppen ersetzt. Die analoge Reaktion mit tert-Butylamin führt nur zum Austausch eines Chloratoms. Die Isomeren von 13 sind durch wiederholte Kristallisation aus n-Hexan trennbar.

Spiroverbindungen vier- und fünfgliedriger $\mathrm{Si}-\mathrm{N}$-Ringe sind in der Literatur bereits mehrfach beschrieben [6]. Wir stellen hier die Synthese einer fünf-vierfünfgliedrigen Spiroverbindung durch Reaktion von 4 mit elementarem Lithium vor, die unter $\mathrm{Si}-\mathrm{Si}$-Bindungsknüpfung und Expansion zweier Vierringe verläuft.

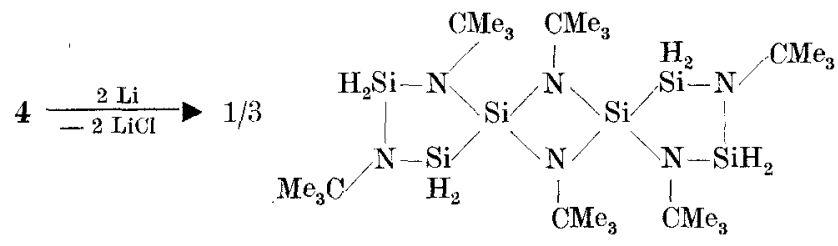

17

Spektroskopische Untersuchungen

Zur Strukturaufklärung der Verbindungen $\mathbf{1 - 1 7}$ wurden außer den Elementaranalysen, NMR- und Massenspektren herangezogen. Die Molekulargewichte wurden massenspektroskopisch bestimmt.

Die den NMR-Spektren zu entnehmenden Parameter sind in Tab. 1 verzeichnet. Die cis- und trans-Isomeren von 11 treten in den ${ }^{1} \mathrm{H}$ - und ${ }^{19} \mathrm{~F}-\mathrm{NMR}$-Spektren als $\mathrm{AA}^{\prime} \mathrm{XX}$-'Typ auf (Abb. 1).

Die ${ }^{19} \mathrm{~F}-\mathrm{NMR}-\mathrm{Spektren}$ der Isomeren von 11 (X-Teil) zeigen das gleiche Aufspaltungsmuster.

Von 17 wurden durch Kristallisation aus Benzol röntgentaugliche Einkristalle erhalten.

Kristallstruktur von 17. 17 kristallisiert in farblosen Prismen in der Raumgruppe $\mathrm{P} 2_{1} / \mathrm{n}$. Der Spirocyclus besteht aus einem zentralen $\mathrm{Si}-\mathrm{N}$-Vierring und aus zwei dazu senkrecht stehenden $\mathrm{Si}_{3}-\mathrm{N}_{2}$-Fünfringen. Im Schwerpunkt des Vierringes befindet sich ein Inversionszentrum. Die $\mathrm{Si}-\mathrm{N}$-Bindungen im Vierring $(173,3(4)$ pm bzw. 173,7(4) pm) sind innerhalb der Fehlergrenzen identisch. Die Si-N-Bindungslängen im Fünfring variieren von 171,2(4) pm $(\mathrm{Si}(3)-\mathrm{N}(2))$ bis $174,1(3) \mathrm{pm}(\mathrm{Si}(1)-\mathrm{N}(1))$, liegen aber damit im normalen Bereich für Cyclosilazane. Die Si-Si-Bindungslänge ist mit 233,1(2) pm vergleichbar mit den $\mathrm{Si}-\mathrm{Si}$-Abständen in anderen Spirocyclen [7]. Die N(3)-C(3)-Bindung 
Tabelle 1 Chemische Verschiebungen der Verbindungen 1-17

\begin{tabular}{|c|c|c|c|c|}
\hline Lfd. $\mathrm{Nr}$. & $\delta^{1} \mathrm{H}[\mathrm{ppm}]$ & $\delta^{13} \mathrm{C}[\mathrm{ppm}]$ & $\delta^{29} \mathrm{Si}[\mathrm{ppm}]$ & $J_{\mathrm{HSi}}[\mathrm{Hz}], \mathrm{J}_{\mathrm{HH}}[\mathrm{H} z z]$ \\
\hline 1 & $\begin{array}{l}1,33 \mathrm{CH}_{3} \\
2,15 \mathrm{NH}\end{array}$ & $\begin{array}{ll}32,39 & \mathbf{C C}_{3} \\
51,79 & \mathbf{C C}_{3}\end{array}$ & $-30,00$ & \\
\hline 2 & $\begin{array}{l}1,29 \mathrm{CH}_{3} \\
5,61 \mathrm{SiH}\end{array}$ & & $-26,03$ & ${ }^{3} \mathrm{~J} H S i N H=4,5$ \\
\hline 3 & $\begin{array}{lll}0,21 & \mathrm{SiCH}_{3} \\
0,88 & \mathrm{CCH}_{3} \\
1,80 & \mathrm{CH} \\
2,79 & \mathrm{CH}_{2} \\
5,6 & \mathrm{SiH}\end{array}$ & & $\begin{array}{r}-19,85 \mathrm{SiH} \\
10,58 \mathrm{SiCH}_{3}\end{array}$ & ${ }^{8} \mathfrak{J}_{\mathrm{HH}}=7,2$ \\
\hline cis-4 & $\begin{array}{l}1,28 \mathrm{CH}_{\mathrm{a}} \\
5,87 \mathrm{SiH}\end{array}$ & $\begin{array}{l}33,03 \mathrm{CC}_{3} \\
50,83 \mathrm{NC}\end{array}$ & $-33,37$ & $\begin{aligned} \mathrm{J}_{\mathrm{HSi}} & =301 \\
{ }^{3} \mathrm{~J}_{\mathrm{HSi}} & =5,5\end{aligned}$ \\
\hline trans $-\overline{4}$ & $\begin{array}{l}1,27 \mathrm{CH}_{3} \\
5,74 \mathrm{SiH}\end{array}$ & $\begin{array}{ll}33,0 & \mathrm{CC}_{3} \\
506,1 & \mathrm{NC}\end{array}$ & $-37,87$ & $J_{\mathrm{HSi}}=303$ \\
\hline 5 & $\begin{array}{l}0,36 \mathrm{SiCH}_{2} \\
1,48 \mathrm{CCH}_{3}\end{array}$ & & $\begin{array}{r}-13,00 \mathrm{SiCl} \\
5,90 \mathrm{SiCH}_{3}\end{array}$ & \\
\hline 6 & $\begin{array}{ll}1,25 \mathrm{CH}_{3} \\
1,89 \mathrm{NH}\end{array}$ & & $-16,81$ & \\
\hline 7 & $\begin{array}{l}0,94 \mathrm{CH}_{3} \\
1,88 \quad \mathbf{C H} \\
2,89 \quad \mathrm{CH}_{2} \\
5,23 \quad \mathrm{SiH}\end{array}$ & & $-20,99$ & \\
\hline 8 & $0,16 \mathrm{CH}_{3}$ & & $\begin{aligned}-11,44 \mathrm{SiCl} \\
7,58 \mathrm{SiMe}_{3}\end{aligned}$ & \\
\hline 9 & & $\begin{array}{l}32,47 \mathrm{CC}_{3} \\
52,98 \mathrm{NC}\end{array}$ & $-43,35$ & ' \\
\hline cig-10 & $1,1: 1 \mathrm{CH}_{\mathrm{s}}$ & & $-24,04$ & \\
\hline trans -10 & $1,05 \mathrm{CH}_{3}$ & & $-25,31$ & \\
\hline cis-11 & $\begin{array}{l}1,22 \mathrm{CH}_{3} \\
5,26 \mathrm{SiH}\end{array}$ & $\begin{array}{l}33,38 \mathrm{CC}_{3} \\
49,70 \mathrm{NC}\end{array}$ & $-44,00$ & ${ }^{4} \mathrm{~J}(\mathbf{H S i N S i H})=5,58$ \\
\hline trans-11 & $\begin{array}{l}1,23 \mathbf{C H}_{3} \\
4,99 \mathrm{SiH}\end{array}$ & $\begin{array}{l}33,65 \mathrm{CC}_{3} \\
49,68 \mathrm{NC}\end{array}$ & $-51,15$ & ${ }^{4} \mathrm{~J}(\mathbf{H S i N S i H})=1,27$ \\
\hline cis-12 & $\begin{array}{ll}1,21 & \mathrm{CCH}_{3} \\
3,52 & \mathrm{OCH}_{3} \\
5,00 \mathrm{SiH}\end{array}$ & $\begin{array}{ll}32,71 & \mathrm{OC} \\
33,31 & \mathrm{CC}_{3} \\
48,76 & \mathrm{NC}\end{array}$ & $-45,00$ & \\
\hline trans-12 & $\begin{array}{l}1,22 \mathrm{CCH}_{3} \\
3,59 \mathrm{OCH}_{3} \\
4,75 \mathrm{SiH}\end{array}$ & $\begin{array}{ll}32,96 & \mathrm{OC} \\
33,62 & \mathrm{CC}_{3} \\
48,91 & \mathrm{NC}\end{array}$ & $-50,55$ & \\
\hline cis-13 & $\begin{array}{l}1,15 \mathrm{CCH}_{3} \\
5,39 \mathrm{SiH} \\
6,85-7,06 \quad \mathrm{C}_{6} \mathrm{H}_{5}\end{array}$ & $\begin{array}{rl}33,23 & \mathrm{CC}_{3} \\
49,68 & \mathrm{NC} \\
119,71 & 3,5-\mathrm{C}_{6} \\
122,04 & 4-\mathrm{C}_{6} \\
129,47 & 2,6-\mathrm{C}_{6} \\
154,12 & \mathrm{O}-\mathrm{C}\end{array}$ & $-48,84$ & \\
\hline trans-13 & $\begin{array}{l}1,19 \mathrm{CH}_{3} \\
5,16 \mathrm{SiH} \\
6,8-7,4 \mathrm{C}_{6} \mathrm{H}_{s}\end{array}$ & $\begin{array}{rl}33,03 & \mathrm{CC}_{3} \\
48,82 & \mathrm{NC} \\
119,81 & 3,5-\mathrm{C}_{8} \\
121,37 & 4-\mathrm{C}_{8} \\
129,26 & 2,6-\mathrm{C}_{8} \\
154,40 & \mathrm{OC}\end{array}$ & $-54,70$ & \\
\hline
\end{tabular}


Tabelle 1 (Fortsetzung)

\begin{tabular}{|c|c|c|c|c|c|}
\hline Lfd. Nr. & $\delta^{1} \mathrm{H}[\mathrm{ppm}]$ & $\delta^{13} \mathrm{C}[\mathrm{ppm}]$ & & $\delta^{28} \mathrm{Si}\{\mathrm{ppm}\}$ & $\mathbf{J}_{\mathrm{HSi}}[\mathrm{Hz}], \mathrm{J}_{\mathrm{HHH}}[\mathrm{Hz}]$ \\
\hline cis-14 & $\begin{array}{l}1,09 \mathrm{CCH}_{3} \\
2,49 \mathrm{NCH}_{9} \\
4,87 \mathrm{SiH}\end{array}$ & $\begin{array}{l}33,14 \mathrm{CC}_{3} \\
35,78 \mathrm{NC}_{2} \\
49,33 \mathrm{NC}\end{array}$ & & $-38,48$ & \\
\hline trans-14 & $\begin{array}{l}1,14 \mathrm{CCH}_{3} \\
2,56 \mathrm{NCH}_{3} \\
4,62 \mathrm{SiH}\end{array}$ & $\begin{array}{ll}33,44 & \mathrm{CC}_{3} \\
36,11 & \mathrm{NC}_{2} \\
48,66 & \mathrm{NC}\end{array}$ & & $-43,00$ & \\
\hline eis-15 & $\begin{array}{l}1,09 \mathrm{CH}_{2} \mathbf{C H}_{3} \\
1,21 \mathrm{C}\left(\mathrm{CH}_{3}\right)_{3} \\
2,93 \mathrm{CH}_{2} \\
4,88 \mathrm{SiH}\end{array}$ & & & $-39,70$ & \\
\hline trans-15 & $\begin{array}{l}1,06 \mathrm{CH}_{2} \mathrm{CH}_{3} \\
1,16{\mathrm{C}\left(\mathrm{CH}_{3}\right)_{3}} \\
2,98 \mathrm{CH}_{2} \\
4,64 \mathrm{SiH}\end{array}$ & & & $-44,96$ & \\
\hline $\operatorname{cis}-16$ & $\begin{array}{l}1,27 \mathrm{C}\left(\mathrm{CH}_{3}\right)_{3} \\
\left.1,29 \mathrm{NHC}_{(\mathrm{CH}}\right)_{3} \\
5,04 \mathrm{SiHNH} \\
5,73 \mathrm{SiHCl}\end{array}$ & $\begin{array}{l}33,50 \mathrm{CC}_{3} \\
33,60 \mathrm{NHCC}_{3} \\
50,0 \mathrm{CC}_{3} \\
50,2 \mathrm{NHC}\end{array}$ & & $\begin{array}{l}-47,11 \mathrm{SiN} \\
-37,09 \mathrm{SiCl}\end{array}$ & $\begin{array}{l}\text { J } \mathrm{HSiNH}=4,5 \\
{ }^{\mathrm{HSiNSiH}}=4,5\end{array}$ \\
\hline trans-16 & $\begin{array}{l}\text { 1,27 } \mathrm{C}\left(\mathrm{CH}_{3}\right)_{3} \\
\left.1,29 \mathrm{NHC}_{\mathrm{CH}}\right)_{3} \\
4,85 \mathrm{SiHNH} \\
5,61 \mathrm{SiHCl}\end{array}$ & $\begin{array}{l}33,50 \mathrm{CC}_{3} \\
33,60 \mathrm{NHCC}_{3} \\
50,0 \mathrm{CC}_{3} \\
50,2 \mathrm{NHC}\end{array}$ & & $\begin{array}{l}-51,49 \mathrm{SiNH} \\
-42,01 \mathrm{SiHCl}\end{array}$ & ${ }^{9} J_{1 I S I N H}=3,7$ \\
\hline 17 & & $\left.\begin{array}{l}\begin{array}{l}30,59 \\
32,04 \\
34,36\end{array} \\
51,24 \\
52,12 \\
56,66\end{array}\right\} \mathrm{CC}_{8}$ & & $\left.\begin{array}{l}-59,4 \\
-35,72 \\
-32,18\end{array}\right\} \mathrm{SiH}_{2}$ & \\
\hline & $\delta^{19} \mathrm{~F}[\mathrm{ppm}]$ & $\mathbf{J}_{\mathrm{HF}}[\mathbf{H z}]$ & $J_{F E}[\mathrm{~Hz}]$ & $\mathbf{J}_{\mathrm{SiF}}[\mathrm{Hz}]$ & $\mathrm{J}_{\mathrm{OE}}[\mathrm{Hz}]$ \\
\hline cis-11 & 39,45 & $\begin{array}{l}{ }^{2} \mathrm{~J}=110,12 \\
{ }^{4} \mathrm{~J}=-1,53\end{array}$ & 13,75 & $\begin{array}{r}\mathbf{J}=317, \mathbf{6} \\
\mathbf{J}=\quad 8.1\end{array}$ & $\begin{array}{l}\cdot y=1,81 \\
y=0,73\end{array}$ \\
\hline trans-11 & 48,41 & $\begin{array}{l}{ }^{2} \mathrm{~J}=108,59 \\
\boldsymbol{J}=11,63\end{array}$ & 1,27 & 326,7 & $\begin{array}{l}{ }^{3} \mathrm{~J}=1,41 \\
{ }^{4} \mathrm{~J}=0,67\end{array}$ \\
\hline
\end{tabular}

liegt in der Ebene des planaren Vierrings. Beide tert.-Butylgruppen stehen ideal gestaffelt zueinander. Zwei Methylgruppen eines tert.-Butyl-Substituenten der Fünfringe $\left(C(11)\right.$ und $\left.C\left(11^{\prime}\right)\right)$ stehen oberhalb und unterhalb des $\mathrm{Si}-\mathrm{N}$-Vierrings.

Alle Bindungslängen und -winkel sind den Tab. 2 u. 3 zu entnehmen. 


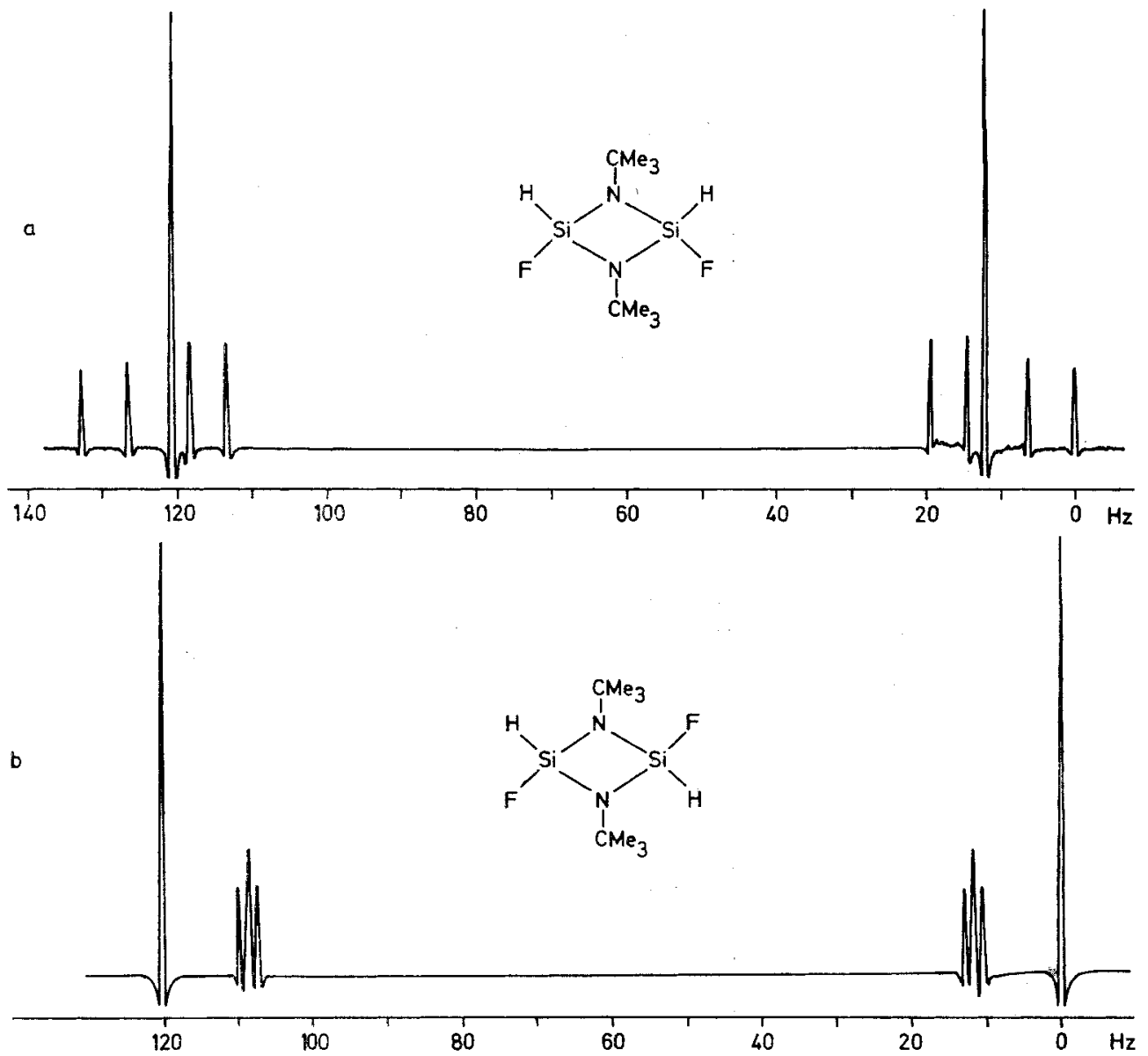

Abb. 1 a) ${ }^{1}$ H-NMR-Spektrum von cis-11 (A-Teil); b) ${ }^{1}$ H-NMR-Spektrum von trans-11 (A-Teil)

Tabelle 2 Bindungslängen $(\mathrm{pm})$ von $\mathbf{1 7}$

\begin{tabular}{llll}
\hline $\mathrm{Si}(1)-\mathrm{Si}(2)$ & $233,1(2)$ & $\mathrm{Si}(1)-\mathrm{N}(1)$ & $174,1(3)$ \\
$\mathrm{Si}(1)-\mathrm{N}(3)$ & $173,7(4)$ & $\mathrm{Si}(1)-\mathrm{Si}(1 \mathrm{a})$ & $253,3(3)$ \\
$\mathrm{Si}(1)-\mathrm{N}(3 \mathrm{a})$ & $173,3(4)$ & $\mathrm{Si}(2)-\mathrm{N}(2)$ & $172,7(4)$ \\
$\mathrm{Si}(3)-\mathrm{N}(2)$ & $171,2(4)$ & $\mathrm{Si}(3)-\mathrm{N}(1)$ & $173,7(4)$ \\
$\mathrm{N}(2)-\mathrm{C}(2)$ & $149,4(7)$ & $\mathrm{N}(1)-\mathrm{C}(1)$ & $152,8(6)$ \\
$\mathrm{C}(1)-\mathrm{C}(11)$ & $152,5(7)$ & $\mathrm{C}(1)-\mathrm{C}(12)$ & $153,4(7)$ \\
$\mathrm{C}(1)-\mathrm{C}(13)$ & $152,4(7)$ & $\mathrm{C}(2)-\mathrm{C}(21)$ & $153,2(8)$ \\
$\mathrm{C}(2)-\mathrm{C}(22)$ & $152,8(9)$ & $\mathrm{C}(2)-\mathrm{C}(23)$ & $152,1(8)$ \\
$\mathrm{N}(3)-\mathrm{C}(3)$ & $146,9(6)$ & $\mathrm{N}(3)-\mathrm{Si}(1 \mathrm{a})$ & $173,3(4)$ \\
$\mathrm{C}(3)-\mathrm{C}(31)$ & $152,4(7)$ & $\mathrm{C}(3)-\mathrm{C}(32)$ & $152,9(8)$ \\
$\mathrm{C}(3)-\mathrm{C}(33)$ & $150,6(8)$ & & \\
\hline
\end{tabular}


Tabelle 3 Bindungswinkel $\left({ }^{\circ}\right)$ von 17

\begin{tabular}{lrlr}
\hline $\mathrm{Si}(2)-\mathrm{Si}(1)-\mathrm{N}(1)$ & $96,7(1)$ & $\mathrm{Si}(2)-\mathrm{Si}(1)-\mathrm{N}(3)$ & $116,7(1)$ \\
$\mathrm{N}(1)-\mathrm{Si}(1)-\mathrm{N}(3)$ & $120,2(2)$ & $\mathrm{Si}(2)-\mathrm{Si}(1)-\mathrm{Si}(1 \mathrm{a})$ & $128,0(1)$ \\
$\mathrm{N}(1)-\mathrm{Si}(1)-\mathrm{Si}(1 \mathrm{a})$ & $135,3(1)$ & $\mathrm{N}(3)-\mathrm{Si}(1)-\mathrm{Si}(1 \mathrm{a})$ & $43,1(1)$ \\
$\mathrm{Si}(2)-\mathrm{Si}(1)-\mathrm{N}(3 \mathrm{a})$ & $116,7(1)$ & $\mathrm{N}(1)-\mathrm{Si}(1)-\mathrm{N}(3 \mathrm{a})$ & $122,3(2)$ \\
$\mathrm{N}(3)-\mathrm{Si}(1)-\mathrm{N}(3 \mathrm{a})$ & $86,2(2)$ & $\mathrm{Si}(1)-\mathrm{Si}(2)-\mathrm{N}(2)$ & $99,4(1)$ \\
$\mathrm{N}(2)-\mathrm{Si}(3)-\mathrm{N}(1)$ & $110,3(2)$ & $\mathrm{Si}(2)-\mathrm{N}(2)-\mathrm{Si}(3)$ & $115,8(2)$ \\
$\mathrm{Si}(2)-\mathrm{N}(2)-\mathrm{C}(2)$ & $122,2(3)$ & $\mathrm{Si}(3)-\mathrm{N}(2)-\mathrm{C}(2)$ & $121,2(3)$ \\
$\mathrm{Si}(1)-\mathrm{N}(1)-\mathrm{Si}(3)$ & $116,5(2)$ & $\mathrm{Si}(1)-\mathrm{N}(1)-\mathrm{C}(1)$ & $127,4(3)$ \\
$\mathrm{Si}(3)-\mathrm{N}(1)-\mathrm{C}(1)$ & $115,9(3)$ & $\mathrm{N}(1)-\mathrm{C}(1)-\mathrm{C}(11)$ & $111,1(4)$ \\
$\mathrm{N}(1)-\mathrm{C}(1)-\mathrm{C}(12)$ & $109,8(4)$ & $\mathrm{N}(1)-\mathrm{C}(1)-\mathrm{C}(13)$ & $110,0(4)$ \\
$\mathrm{N}(2)-\mathrm{C}(2)-\mathrm{C}(21)$ & $108,9(4)$ & $\mathrm{N}(2)-\mathrm{C}(2)-\mathrm{C}(22)$ & $108,8(4)$ \\
$\mathrm{N}(2)-\mathrm{C}(2)-\mathrm{C}(23)$ & $111,9(4)$ & $\mathrm{Si}(1)-\mathrm{N}(3)-\mathrm{C}(3)$ & $132,7(3)$ \\
$\mathrm{Si}(1)-\mathrm{N}(3)-\mathrm{Si}(1 \mathrm{a})$ & $93,8(2)$ & $\mathrm{C}(3)-\mathrm{N}(3)-\mathrm{Si}(1 \mathrm{a})$ & $133,2(3)$ \\
$\mathrm{N}(3)-\mathrm{C}(3)-\mathrm{C}(31)$ & $113,1(4)$ & $\mathrm{N}(3)-\mathrm{C}(3)-\mathrm{C}(32)$ & $110,7(4)$ \\
$\mathrm{N}(3)-\mathrm{C}(3)-\mathrm{C}(33)$ & $110,1(4)$ & & \\
\hline
\end{tabular}

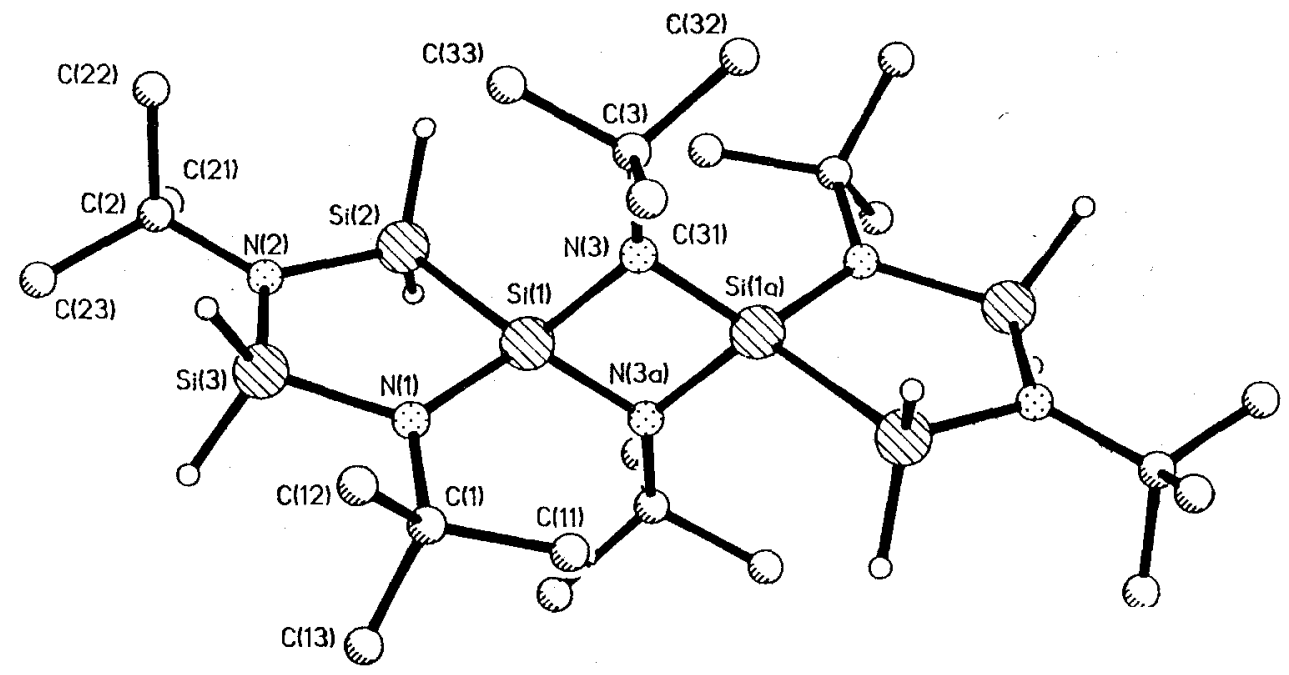

Abb. 2 Molekülstruktur von 1:

\section{Experimenteller Teil}

Alle Umsetzungen wurden unter Ausschluß von Luftfeuchtigkeit unter Inertgas durehgeführt. Die dargestellten Verbindungen sind farblos. Elementaranalysen s. Tab. 4. 1-16 destillieren ohne Zersetzung. NMR-Spektren (30\%ige Lösungen in $\mathrm{CDCl}_{3}$; TMS, $\mathrm{C}_{6} \mathrm{~F}_{6}$ int.); Bruker WP $80 \mathrm{SY}$ bzw. AM 250 Kernresonanzgeräte. Massenspektren CH5-Spektrometer, Varian. IR-Spektren Perkin-Elmer Gitterspektrometer 325 .

Kristallstrukturuntersuchung. Zur Datensammlung wurde ein Stoe-Siemens-AED-Diffraktometer mit graphitmonochromatisierter MoK $\alpha$-Strahlung $(\lambda=71,069 \mathrm{pm})$ benutzt. Es wurden 2338 Reflexe im Bereich $7^{\circ}<2 \Theta<45^{\circ}$ bei Raumtemperatur gemessen. Die Struktur wurde mit direkten Methoden (SHELXTL) gelöst. Zur Verfeinerung wurden 1526 Reflexe mit $\underline{F}>4 \sigma(\underline{F})$ 
benutzt. Die Methylwasserstoffatome wurden gèmäß ihrer idealen Geometrie positioniert (C-HAbstand $96 \mathrm{pm}, \mathrm{H}-\mathrm{C}-\mathrm{H}$-Winkel $109,9^{\circ}, \underline{\mathrm{U}}(\mathrm{H})=1,5 \underline{\mathrm{U}}_{\mathrm{eq}}(\mathrm{C})$, wobei $\underline{\mathrm{U}}_{\mathrm{eq}}(\mathrm{C})=1 / 3$ der Spur des orthogonalen $\underline{\mathrm{U}}_{\mathrm{ij}}$-Tensors). Analoges gilt für die $\mathrm{Si}-\mathrm{H}$-Wasserstoffatome. Die $\mathrm{Si}-\mathrm{H}$-Bindung wurde auf $140 \mathrm{pm}$ gesetzt, da Si-H-Bindungen. im Röntgenbeugungsexperiment etwas kürzer erscheinen als im Elektronenbeugungsexperiment [8]. Für den Thermalparameter gilt $\mathrm{U}(\mathrm{H})=1,2 \underline{\mathrm{U}}_{\mathrm{eq}}(\mathrm{Si})$.

Kristallographische Daten

$\begin{array}{ccll}\text { Raumgruppe }: & \mathrm{P} 2_{1} / \mathbf{n} & \mathrm{V} & 1,8015 \mathrm{~nm}^{3} \\ \mathrm{Z} & 2 & \mu(\mathbf{M o K} \alpha) & 0,248 \mathrm{~mm}^{-1} \\ \mathrm{a} & 902,3(4) \mathrm{pm} & \mathrm{R} & 0,0599 \\ \mathrm{~b} & 1189,6(2) \mathrm{pm} & \mathrm{R}_{\mathrm{w}} & 0,0487 \\ \mathrm{e} & 1691,2(3) \mathrm{pm} & \mathrm{w}^{-1} & \left(\sigma^{2}(\mathbf{F})+\mathrm{gF}^{2}\right) \\ \beta & 97,09(3)^{\circ} & \mathrm{g} & 0,000366 \text { (optimiert) }\end{array}$

Tabelle 4 Elementaranalysen der Verbiudungen 1-17

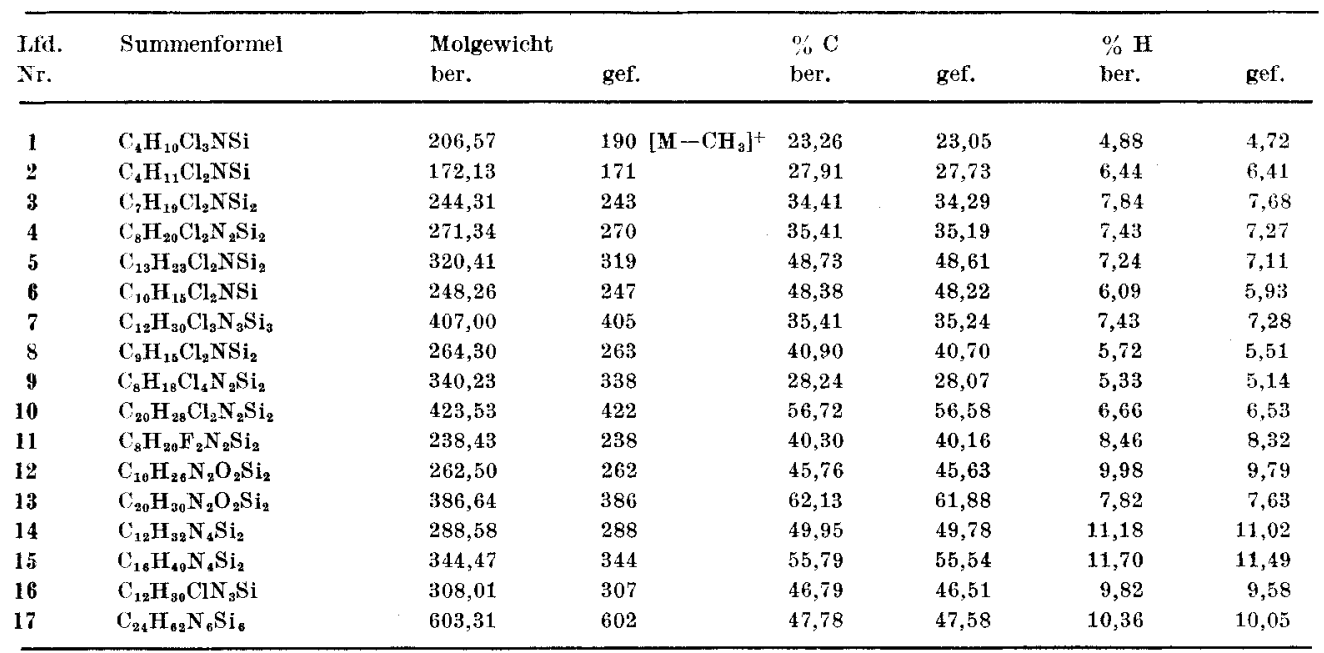

Verbind ungen 1-6. 0,2 mol LiNHCMe $3(1,2), \operatorname{LiN}\left(\mathrm{CH}_{2} \mathrm{CHMe}_{2}\right) \mathrm{SiMe}_{3}(\mathbf{3})$ bzw. $\mathrm{LiN}\left(\mathrm{CMe}_{3}\right) \mathrm{SiMe}_{3}$ in $100 \mathrm{ml} \mathrm{n}$-Hexan und $20 \mathrm{ml}$ THF werden langsam unter Rühren bei $0^{\circ} \mathrm{C}$ zu einer Lösung des jeweiligen Chlorsilans in $100 \mathrm{ml} \mathrm{n}$-Hexan und $20 \mathrm{ml}$ THF getropft. Anschließend wird das Reaktionsgemisch $2 \mathrm{~h}$ am Rückfluß erhitzt und das Rohprodukt von den Lösungsmitteln und dem ausgefallenen LiCl abgetrennt. 1-6 werden destillativ gereinigt. 5 kristallisiert nach der Destillation aus und wird aus $\mathrm{n}$-Hexan umkristallisiert.

tert-Butylamino-trichlorsilan 1. Ausb. $36,7 \mathrm{~g}(98 \%)$; Sdp. $41^{\circ} \mathrm{C} / 6 \mathrm{mbar}$; MS m/e $=190(100)$ $[\mathrm{M}-\mathrm{Me}]^{+}$; IR (kapillar) $3390 \mathrm{~cm}^{-1}(\mathrm{NH})$.

tert-Butylamino-diehlorsilan 2. Ausb. $15,4 \mathrm{~g}(45 \%)$; Sdp. $45^{\circ} \mathrm{C} / 50 \mathrm{mbar}$; MS m/e $=171$ (35) $\left[\mathrm{M}^{+}\right], 156(100)[\mathrm{M}-\mathrm{Me}]^{+}$; IR (kapillar) $3395 \mathrm{~cm}^{-1}(\mathrm{NH}), 2235 \mathrm{~cm}^{-1}(\mathrm{SiH})$.

Isobutyl(trimethylsilyl)amino-diehlorsilan 3. Ausb. $21,0 \mathrm{~g} \quad(43 \%)$; Sdp. $89^{\circ} \mathrm{C} / 18 \mathrm{mbar}$; MS m/e $=243(3)\left[\mathrm{M}^{+}\right], 200(100)\left[\mathrm{M}-\mathrm{C}_{3} \mathrm{H}_{7}\right]^{+} ; \mathrm{IR}$ kapillar) $2240 \mathrm{~cm}^{-1}(\mathrm{SiH})$.

1,3-Di-tert-butyl-2,4-dichlor-2,4-dihydrido-cyclodisilazan 4. Ausb. 26,6 g (87\%); Sdp. $32{ }^{\circ} \mathrm{C} / 0,01 \mathrm{mbar} ; \mathrm{MS} \mathrm{m} / \mathrm{e}=270(2)\left[\mathrm{M}^{+}\right], 255(100)[\mathrm{M}-\mathrm{Me}]^{+} ; \mathrm{IR}$ (kapillar) $2200 \mathrm{~cm}^{-1}(\mathrm{SiH})$. 
tert-Butyl(trimethylsilyl) amino-dichlor-phenylsilan 5. Ausb. $18,6 \mathrm{~g}(29 \%) ; \mathrm{Sdp} .105^{\circ} \mathrm{C} /$ 0,01 mbar; Sehmp. $64^{\circ} \mathrm{C}$; MS m/e $=319(100)\left[\mathrm{M}^{+}\right]$.

tert-Butylamino-dichlor-phenylsilan 6. Ausb. $29,7 \mathrm{~g} \quad(60 \%) ; \mathrm{Sdp} .60^{\circ} \mathrm{C} / 0,01 \mathrm{mbar} ; \mathrm{MS}$ $\mathrm{m} / \mathrm{e}=247(5)\left[\mathrm{M}^{+}\right], 232(100)[\mathrm{M}-\mathrm{Me}]^{+}$; IR (kapillar) $3385 \mathrm{~cm}^{-1}(\mathrm{NH})$.

1,3,5-Tri-iso-butyl-2,4,6-trichlor-2,4,6-trihydridocyclotrisilazan 7 . $0,1 \mathrm{~mol} 3$ und $0,1 \mathrm{~g}$ $\mathrm{Al}_{2} \mathrm{Cl}_{6}$ werden ohne Lösungsmittel etwa $5 \mathrm{~h}$ unter Rückfluß erhitzt. Anschließend wird das abgespaltene $\mathrm{Me}_{3} \mathrm{SiCl}$ im Vakuum abgezogen und 7 durch Kristallisation aus n-Hexan gereinigt. Ausb. $7,3 \mathrm{~g}(54 \%) ;$ MS m/e $=405(100)\left[\mathrm{M}^{+}\right]$; IR (Nujol) $2205 \mathrm{~cm}^{-1}(\mathrm{SiH})$.

(Trimethylsilyl)amino-dichlor-phenylsilan 8. Darstellung analog 7 und 5 Reinigung destillativ. Ausb. $19,0 \mathrm{~g}(72 \%) ; \mathrm{MS} \mathrm{m} / \mathrm{e}=263(100)\left[\mathrm{M}^{+}\right]$; IR (kapillar) $3370 \mathrm{~cm}^{-1}(\mathrm{NH})$.

\section{Verbindungen 9 und 10}

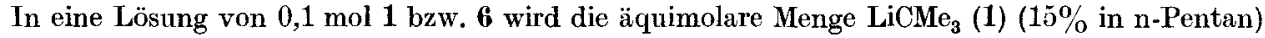
bzw. Lin- $\mathrm{C}_{4} \mathrm{H}_{9}(6)(15 \%$ ig in n-Hexan) getropft. Anschließend wird $2 \mathrm{~h}$ unter Rückfluß erhitzt, das ausgefallene $\mathrm{LiCl}$ abgefrittet und die Cyclodisilazane aus n-Hexan umkristallisiert.

1,3-Di-tert-butyl-2,2,4,4-tetrachlor-cyclodisilazan 9. Ausb. $8,8 \mathrm{~g}(52 \%)$; Sehmp. $106^{\circ} \mathrm{C}$; MS $\mathrm{m} / \mathrm{e}=338(60)\left[\mathrm{M}^{+}\right]$.

1,3-Di-tert-butyl-2,4-dichlor-2,4-diphenyleyclodisilazan 10. Ausb. 14,3 g (67\%); Schmp. $104^{\circ} \mathrm{C} ; \mathrm{MS} \mathrm{m} / \mathrm{e}=422(10)\left[\mathrm{M}^{+}\right], 407(100)[\mathrm{M}-\mathrm{Me}]^{+}$.

1,3-Di-tert-butyl-2,4-difluor-2,4-dihydridocyclodisilazan 11. $0,05 \mathrm{~mol} 4$ werden mit einer Aufschlämmung von $0,11 \mathrm{~mol} \mathrm{NaF}$ in $50 \mathrm{ml} \mathrm{CH}_{3} \mathrm{CN} 4 \mathrm{~h}$ am Rückfluß erhitzt. Anschließend wird das Lösungsmittel angezogen und 11 destillativ gereinigt.

Ausb. $5,9 \mathrm{~g}(42 \%) ; \mathrm{Sdp} .72^{\circ} \mathrm{C} / 20 \mathrm{mbar} ; \mathrm{MS}: \mathrm{m} / \mathrm{e}=238(2)\left[\mathrm{M}^{+}\right], 223(100)[\mathrm{M}-\mathrm{Me}]^{+}$; IR (kapillar): $2205 \mathrm{~cm}^{-1}(\mathrm{SiH})$.

1,3-Di-tert-butyl-2,4-dihydrido-2,4-dimethoxy-cyclodisilazan 12. $Z_{u}$ einer Lösung von $0,05 \mathrm{~mol} 4$ und $0,1 \mathrm{~mol} \mathrm{Et}_{3} \mathrm{~N}$ in $100 \mathrm{ml} \mathrm{n}$-Hexan werden unter Rühren $0,1 \mathrm{~mol} \mathrm{MeOH}$ getropft. Anschließend wird $1 \mathrm{~h}$ unter Rückfluß erhitzt, $\mathbf{E t}_{3} \mathrm{~N} \cdot \mathrm{HCl}$ abgefrittet und das Lösungsmittel im Vakuum abgezogen. 12 wird destillativ gereinigt.

Ausb. 7,4 g (57\%); Sdp. $25^{\circ} \mathrm{C} / 0,01 \mathrm{mbar} ; \mathrm{MS} \mathrm{m} / \mathrm{e}=262(3)\left[\mathrm{M}^{+}\right], 247(100)[\mathrm{M}-\mathrm{Me}]^{+}$.

1,3-Di-tert-butyl-2,4-dihydrido-2,4-diphenoxy-cyclodisilazan $13 . \quad 0,01 \mathrm{~mol} 4$ in $50 \mathrm{ml}$ n-Hexan wird mit 0,02 mol LiOC $_{6} \mathrm{H}_{5}$ in $50 \mathrm{ml} \mathrm{THF} \mathrm{versetzt} \mathrm{und} 20 \mathrm{~min}$ unter Rückfluß erhitzt. Die Aufarbeitung erfolgt analog 12. cis 13 kristallisiert aus n-Hexan aus, trans 13 wird destillativ gereinigt.

cis 13. Ausb. 1,2 g (31\%); Schmp. $112^{\circ} \mathrm{C}$; MS m/e $=386(100)\left[\mathrm{M}^{+}\right]$; trans 13. Ausb. $1,7 \mathrm{~g}$ $(45 \%)$; Sdp. $100^{\circ} \mathrm{C} / 0,01 \mathrm{mbar} ; \mathrm{MS}: \mathrm{m} / \mathrm{e}=386(36)\left[\mathrm{M}^{+}\right], 329(48)\left[\mathrm{M}-\mathrm{C}_{4} \mathrm{H}_{9}\right]^{+}$

\section{Verbindungen 14-16}

$0,05 \mathrm{~mol} 4$ in $100 \mathrm{ml} \mathrm{n}$-Hexan werden bei $-15^{\circ} \mathrm{C}(14)$ bzw. $20^{\circ} \mathrm{C}(15,16)$ mit 0,2 mol des jeweiligen Amins versetzt. Aufarbeitung und Reinigung erfolgt analog 12.

1,3-Bis(dimethylamino)-2,4-di-tert-butyl-1,3-dihydridocyclodisilazan 14. Ausb. 8,5 g $(59 \%)$; Sdp. $40^{\circ} \mathrm{C} / 0,01 \mathrm{mbar} ; \mathrm{MS}: \mathrm{m} / \mathrm{e}=288(6)\left[\mathrm{M}^{+}\right], 273(100)[\mathrm{M}-\mathrm{Me}]^{+}$; IR (kapillar) $2140 \mathrm{~cm}^{-1}$ (SiH).

1,3-Bis(diethylamino) -2, 4-di-tert-butyl-1,3-dihydrido-cyclodisilazan 15. Ausb. $9,1 \mathrm{~g}$ (53\%). Sdp. $65^{\circ} \mathrm{C} / 0,01 \mathrm{mbar} ; \mathrm{MS} \mathrm{m} / \mathrm{e}=344(8)[\mathrm{M}]^{+}, 329(100)[\mathrm{M}-\mathrm{Me}]^{+}$; IR (kapillar) $2130 \mathrm{~cm}^{-1}(\mathrm{SiH})$; 
1-tert-Butylamino-2,4-di-tert-butyl-3-chlor-1,3-dihydridocyclodisilazan 16. Ausb. 9,4 g $(61 \%)$; Sdp. $65^{\circ} \mathrm{C} / 0,01 \mathrm{mbar}$; MS m/e $=307(2)[\mathrm{M}]^{+}, 292(100)[\mathrm{M}-\mathrm{Me}]^{+}$; IR (kapillar) $2180 \mathrm{~cm}^{-1}$ (SiH), $3380 \mathrm{~cm}^{-1}(\mathrm{NH})$.

\section{$1,3,6,8,10,12$-Hexa-tert-butyl-2,2,4,4,9,9,11,11-octahydrido-1,3,6,8,10,12-hexa-aza-} $\mathbf{2 , 4 , 5 , 7 , 9 , 1 1 - h e x a s i l a - d i s p i r o [ 4 . 1 . 4 . 1 ] - d o d e c a n ~ 1 7 . ~} \mathrm{Zu} 0,08 \mathrm{~mol} \mathrm{Li}$-Pulver in $50 \mathrm{ml} \mathrm{THF}$ werden unter Rühren und Eisbadkühlung langsam 0,04 mol 4 getropft. Die Reaktion verläuft exotherm. Nach Abtrennen des ausgefallenen. $\mathrm{LiCl}$ wird 17 aus Benzol umkristallisiert. Ausb. 1,9 g (24\%); Schmp. $273^{\circ} \mathrm{C}$; MS m/e $=602(4)\left[\mathrm{M}^{+}\right], 587(38)[\mathrm{M}-\mathrm{Me}]^{+}, 585(100):$ IR (kapillar) $2110,2160 \mathrm{~cm}^{-1}$ (SiH).

Der Deutschen Forschungsgemeinschaft und dem Fonds der Chemischen Industrie danken wir für die Unterstützung der Arbeit.

\section{Literatur}

[1] Wannagat, U.: Chemiker Ztg. 97 (1973) 105.

[2] Wannagat, U.; Schreiner, G.: Mh. Chem. 96 (1965) 1895.

[3] Harris, D. H.; Lappert, M. F.: J. Organomet. Chem. Library 2 (1976) 13.

[4] Kurnahbied, U.; Neemann, J.; Meller, A.: Z. anorg. allg. Chem. 429 (1977) 63.

[5] Wannagat, U.: Adv. Inorg. Chem. Radiochem. 6 (1964) 225.

[6] Schltngmann, M.; Wannagat, U.: Z. anorg. allg. Chem. 499 (1977) 74.

[7] Wannagat, U.; Klemke, S.; Mootz, D.; Reski, H. D.: J. Organomet. Chem. 178 (1979) 83.

[8] Glidnwerd, C.; Ropiette, A. G.; Sheldrick, G. M.: J. Chem. Soc., Chem. Commun. 1970, 931.

Bei der Redaktion eingegangen am 23. April 1987.

Anschr. d. Verf.: S. Bartholmei, Prof. Dr. U. Ktingebiel, Prof. Dr. G. M. Sheldrick, D. Stalke, Inst. f. Anorg. Chemie d. Univ., Tammannstr. 4, D-3400 Göttingen 